\title{
Antisense oligonucleotide therapy reduces seizures and extends life span in an SCN2A gain-of-function epilepsy model
}

\author{
Melody Li, ${ }^{1}$ Nikola Jancovski, ${ }^{1}$ Paymaan Jafar-Nejad, ${ }^{2}$ Lisseth E. Burbano, ${ }^{1}$ Ben Rollo,, Kay Richards, ${ }^{1}$ Lisa Drew, ${ }^{1}$ Alicia Sedo, ${ }^{1}$ \\ Jacqueline Heighway, ${ }^{1}$ Svenja Pachernegg, ${ }^{1}$ Armand Soriano, ${ }^{2}$ Linghan Jia, ${ }^{1}$ Todd Blackburn, ${ }^{1,3}$ Blaine Roberts, ${ }^{1}$ \\ Alex Nemiroff, ${ }^{3,4}$ Kelley Dalby, ${ }^{3,4}$ Snezana Maljevic, ${ }^{1}$ Christopher A. Reid, ${ }^{1}$ Frank Rigo, ${ }^{2}$ and Steven Petrou ${ }^{1,3,4}$
}

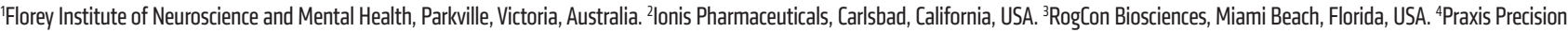
Medicines, Boston, Massachusetts, USA

\begin{abstract}
De novo variation in SCN2A can give rise to severe childhood disorders. Biophysical gain of function in SCN2A is seen in some patients with early seizure onset developmental and epileptic encephalopathy (DEE). In these cases, targeted reduction in SCN2A expression could substantially improve clinical outcomes. We tested this theory by central administration of a gapmer antisense oligonucleotide (ASO) targeting Scn2a mRNA in a mouse model of Scn2a early seizure onset DEE (Q/+ mice). Untreated $\mathrm{Q} /+$ mice presented with spontaneous seizures at $\mathrm{P} 1$ and did not survive beyond $\mathrm{P3O}$. Administration of the ASO to $Q /+$ mice reduced spontaneous seizures and significantly extended life span. Across a range of behavioral tests, Scn2a ASO-treated Q/+ mice were largely indistinguishable from WT mice, suggesting treatment is well tolerated. A human SCN2A gapmer ASO could likewise impact the lives of patients with SCN2A gain-of-function DEE.
\end{abstract}

\section{Introduction}

Developmental and epileptic encephalopathies (DEEs) are devastating neurological disorders presenting during infancy and early childhood (1). The affected children have ongoing, refractory seizures in addition to profound global developmental delay, intellectual disability, and movement disorders (1). The prognosis of these patients is poor, marked by progressive disability and increased risk of early death (1). The limited treatment options, presence of comorbidities, and the need for long-term supported care represent a burden for affected patients, caregivers, and health services.

Only a decade ago little was known about DEE etiologies, but the employment of next-generation sequencing has identified single-gene de novo pathogenic variations as the main cause (2). The number of identified DEE genes is increasing, with those encoding ion channels, as the pivotal brain excitability regulators, being the most represented (2-4). Identification of the genetic cause and increased understanding of the mechanistic basis of DEEs have laid the foundation for the development of precision medicine therapeutic approaches. This is especially important as the current

Related Commentary: https://doi.org/10.1172/JCI155233

Authorship note: $\mathrm{ML}$ and NJ contributed equally to this work.

Conflict of interest: $S$ Petrou, AN, and KD received payments from RogCon and Praxis Precision Medicines. PJN, FR, and A Soriano are employees of Ionis Pharmaceuticals. Copyright: (5) 2021, American Society for Clinical Investigation.

Submitted: June 7, 2021; Accepted: October 1, 2021; Published: December 1, 2021.

Reference information: / Clin Invest. 2021;131(23):e152079.

https://doi.org/10.1172/JCI152079. treatments primarily focus on the symptomatic control of seizures while the developmental aspects of the disease remain untended.

Several major technological advances have enabled the possibility of establishing effective targeted gene therapies for DEEs. After several decades of development, antisense oligonucleotides (ASOs) have recently been approved as a treatment for spinal muscular atrophy and Duchenne muscular dystrophy, which demonstrated that RNA-targeted therapies are a viable therapeutic strategy for these devastating neurogenetic disorders $(5,6)$. ASOs are single-stranded oligonucleotides that are designed to specifically target the RNA of interest through Watson-Crick base pairing, which results in RNase H1-mediated mRNA degradation, modulation of pre-mRNA splicing, or regulation of translational efficiency $(7,8)$.

$S C N 2 A$ encodes the $\alpha$-subunit of a voltage-gated sodium channel $\left(\mathrm{Na}_{\mathrm{v}} 1.2\right)$ and is involved in the initiation and conduction of action potentials, particularly during early postnatal development when its expression is higher than that of other voltagegated sodium channel isoforms $(9,10)$. In recent years, this gene was implicated in a range of neurodevelopmental disorders (11, 12). The phenotypic spectrum associated with $S C N 2 A$ de novo variants includes schizophrenia, autism spectrum disorder, intellectual disability, and a myriad of epileptic syndromes including DEEs (11-15). Case history studies of patients with SCN2A DEE have identified 2 major clinical presentations. Based on the age of seizure onset, pharmacoresponsiveness, and disease severity, we recognize DEE with an early-onset seizures starting within the first 3 months of life and DEE with late-onset seizures commencing between 3 months and 4 years of age (13). Functional inves- 
tigation of a number of $S C N 2 A$ DEE pathogenic variations has shown that the early-onset seizure phenotype is caused by gain of function of $S C N 2 A$ whereas the late-onset seizure DEE is linked to a loss-of-function patho-mechanism $(11,16)$. These studies correlate well with the observation that patients with early onset of seizures respond better to antiseizure drugs that nonselectively block sodium channel function, such as phenytoin (13). Moreover, this observation suggests that patients could be amenable to gene therapy designed to reduce $S C N 2 A$ expression.

To corroborate this hypothesis, we engineered a mouse model of SCN2A DEE with early seizure onset based on the human equivalent of c.5645G $>\mathrm{A}$ variant, which results in the protein change p.R1882Q. This is one of the most recurrent pathogenic variants for this form of SCN2A DEE, with 6 cases reported to date (12). In some of these patients, phenytoin was reported to partially improve seizure outcomes (13). However, within the therapeutic window of phenytoin, seizures are not fully resolved and developmental impairment remains unaddressed. In vitro biophysical analysis confirmed the $S C N 2 A$ p.R1882Q variant causes larger peak and persistent sodium currents, with dynamic clamp simulations predicting a higher action potential firing frequency (16).

Here, an antisense oligonucleotide (ASO) specifically designed to downregulate mouse $S c n 2 a$ mRNA expression ( $S c n 2 a$ ASO) was delivered to the mouse model carrying the R1882Q corresponding variant $(\mathrm{Q} /+)$. The $\mathrm{Q} /+$ mice develop spontaneous seizures at $\mathrm{P} 1$ and suffer premature death, thus recapitulating the severe symptoms observed in patients with early seizure onset DEE. We show that Scn $2 a$ ASO-mediated knockdown effectively prevents premature death and suppresses spontaneous seizures in the Q/+ mouse model. The Scn $2 a$ ASO-treated Q/+ mice behaved similarly to WT $(+/+)$ mice in almost all other motor and psychosocial tests. Scn $2 a$ ASO is the first therapy to specifically address the disease phenotype of $S C N 2 A$ early seizure onset DEE, positioning ASO therapy as a promising strategy for $S C N 2 A$ gain-of-function diseases.

\section{Results}

The SCN2A gain-of-function DEE mouse model. The SCN2A R1882Q variant is located at the C-terminal tail (Figure 1A). Mirroring the clinical presentation of SCN2A p.R1882Q DEE, Q/+ mice have a severe, early seizure onset phenotype. Spontaneous tonic seizures were observed as early as P1, which later developed into tonic-clonic seizures with hindlimb extension (Figure 1B). The earliest death occurred at P13 and the median survival was P18 (Figure 1C). No significant difference in body weight was found between $\mathrm{Q} /+$ and +/+ mice when measured at P10 or P21 (Figure 1D). Because no Q/+ mice survived beyond P30, and they present with frequent seizures, there was a limited opportunity for behavioral characterization.

Dynamic clamp modeling predicted $S C N 2 A$ p.R1882Q to increase action potential firing (16), but this has not been verified in vivo. Thus, whole-cell recording was performed in $\mathrm{L} 2 / 3$ cortical pyramidal neurons in brain slices of $+/+$ and $Q /+$ mice. In comparison to $+/+$ mice, pyramidal neurons from $\mathrm{Q} /+$ mice showed overt gain-of-function phenotype, including higher action potential frequency (Figure 1, E and F). The maximal firing frequency was 15.4 \pm 1.16 and $21.7 \pm 1.32$ in $+/+$ and $Q /+$ neurons, respectively. Fur- thermore, Q/+ neurons showed higher input resistance (Figure $1 \mathrm{G})$ and lower rheobase (Figure $1 \mathrm{H})$.

Scn 2 a ASO reduces Scn $2 a$ mRNA and protein levels. An ASO targeting mouse Scn $2 a$ (GCTCATGTTACTCCTACCCT) was designed to test the hypothesis that reducing $\operatorname{Scn} 2 a$ expression would be therapeutic for $\mathrm{Q} /+$ mice which express the SCN $2 A$ gain-of-function pathogenic variant. The $\operatorname{Scn} 2 a$ ASO was synthesized with five $2^{\prime}$-O-methoxyethyl (MOE) modified nucleotides at each end of the oligonucleotide, and 10 DNA nucleotides in the center. Because we aim to find an ASO therapy for SCN2A gain-of-function variants in general, the $\operatorname{Scn} 2 a$ ASO designed here is not allele specific.

Using a pan-ASO antibody (17), we confirmed broad ASO distribution throughout the brain of mice injected via the intracerebroventricular (i.c.v.) route at P1 (Supplemental Figure 1; supplemental material available online with this article; https:// doi.org/10.1172/JCI152079DS1). Quantification of overlapping ASO and neuronal marker in 10 different brain regions revealed an average of $97.6 \% \pm 0.2 \%$ of neurons examined contained Scn $2 a$ ASO (Supplemental Table 1, $n=4$ mice).

Scn $2 a$ ASO specifically reduced Scn $2 a$ mRNA and had no effect on the other voltage-gated sodium channels (Figure 2A). At the protein level, mass spectrometry determined a $73.9 \%$ reduction in Scn2a protein levels in the Scn2a ASO-treated mice when compared with the control nontargeting ASO group (Figure 2B). Using an anti-Scn $2 a$ protein antibody (Supplemental Figure 2), immunohistochemical analysis also provided qualitative data suggesting that Scn $2 a$ protein expression was reduced along the axonal initial segment and in neuropils (Figure 2C). To determine the effective dose for $50 \%\left(\mathrm{ED}_{50}\right) \operatorname{Scn} 2 a$ mRNA reduction, mice were i.c.v. injected with Scn2a ASO at P1, P15, and P30 and Scn2a mRNA level measured 2 weeks after i.c.v. injection (Supplemental Figure 3). The Scn $2 a$ ASO ED 50 determined for P1, P15, and P30 were $2 \mu \mathrm{g}, 20 \mu \mathrm{g}$, and $33 \mu \mathrm{g}$, respectively.

Scn2a ASO rescues premature death and seizure phenotype in $Q /+$ mice. To test whether the $\operatorname{Scn} 2 a$ ASO can mitigate disease in the $S C N 2 A$ early seizure onset DEE mouse model, we i.c.v. injected Q/+ mice with a single dose of $\operatorname{Scn} 2 a \mathrm{ASO}_{\mathrm{ED}}$ or control ASO at P1. As expected, control ASO did not affect survival and median survival was P20 ( $n=39$ mice, Figure $3 \mathrm{~A})$. In sharp contrast, a single dose of $\operatorname{Scn} 2 a$ ASO ED $_{50}$ at P1 more than doubled the median survival to $\mathrm{P} 47$ ( $n=49$ mice, Figure $3 \mathrm{~A}$ ). At experimental endpoint (P80), 9.63\% of the Scn $2 a$ ASO ED 50 group remained alive.

To determine if survival could be further extended by repeated administration, Q/+ mice were first i.c.v. injected at P1 with Scn2a ASO ED A0 At P27-P30, a second dose of Scn2a ASO $\mathrm{ED}_{50}$ or control ASO was administered. Q/+ mice that received 2 doses of Scn $2 a$ ASO $\mathrm{ED}_{50}$ had significantly longer life span than those redosed with the control ASO (median survival for control ASO: P86, $n=11$; for Scn $2 a$ ASO ED $_{50}$ : P145, $n=15$ ) (Figure 3B). This suggests that repeated dosing in later development maintained the therapeutic level of Scn $2 a$ ASO, effectively suppressing disease reemergence.

In practice, the ASO therapy would be delivered after genetic diagnosis and the DEE symptoms would have likely progressed before initiating ASO treatment. Therefore, it was critical to 
A

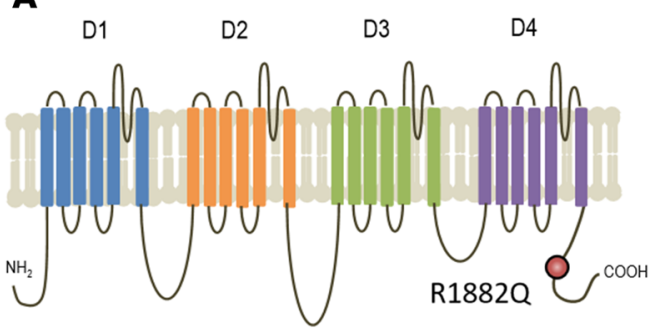

C

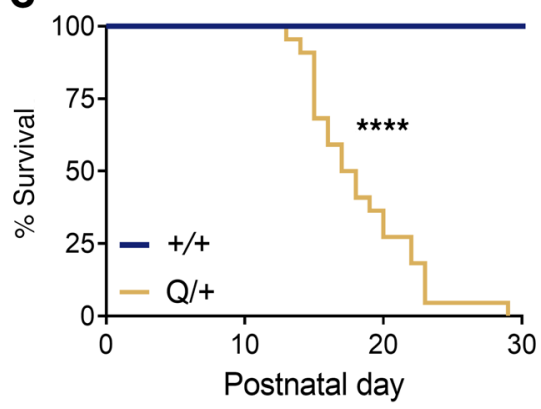

E

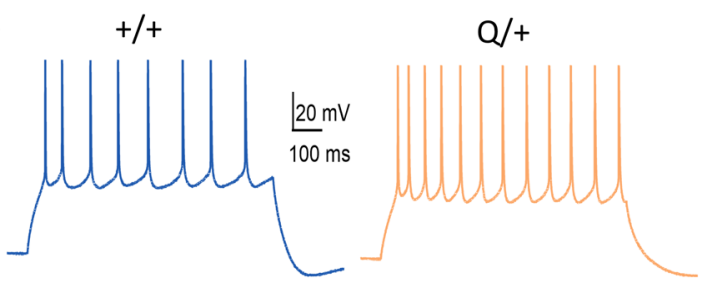

G

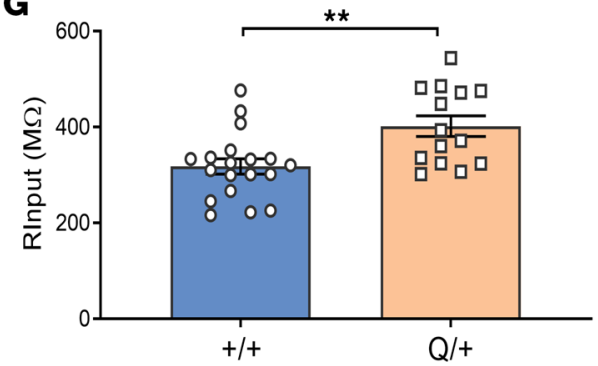

B
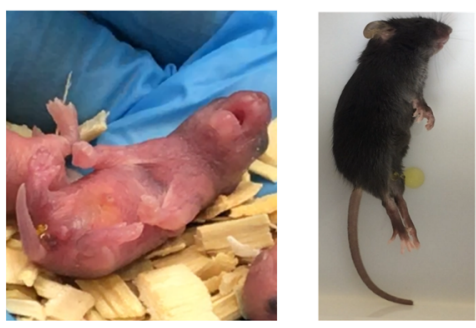

D

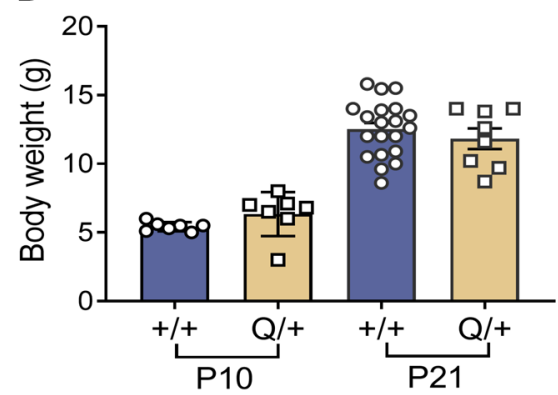

$\mathbf{F}$

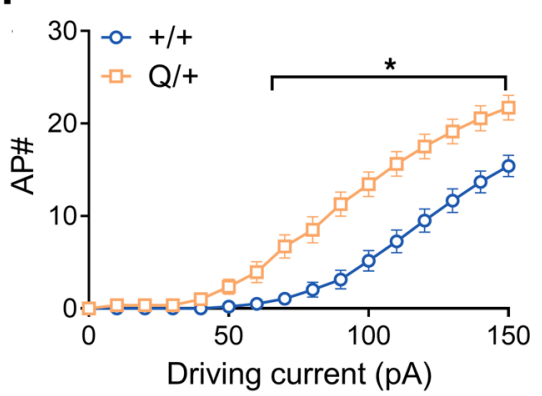

H

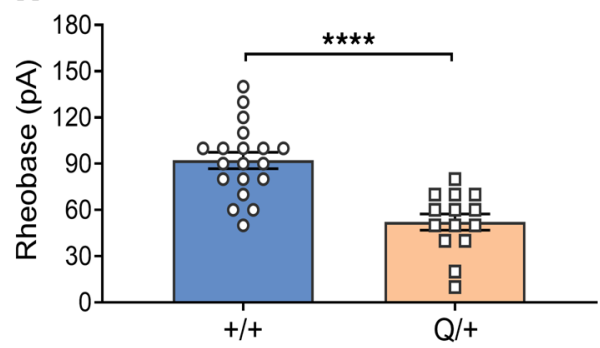

Figure 1. Disease phenotype of the Q/+ mouse model. (A) Schematic presentation of SCN2A channel depicting 4 domains (D1-D4), each comprised of 6 transmembrane regions, and the intracellular $\mathrm{N}$ and $\mathrm{C}$-terminus of the channel. The p.R1882Q variant is predicted to affect the C-terminus of the channel. (B) Images of Q/+ mice undergoing spontaneous seizure at P1 (left) and P25 (right). (C) Survival curves of $\mathrm{Q} /+$ and $+/+$ mice. $(+/+n=19, Q /+n=22) .{ }^{* * * *} P<$ 0.0001 , log rank test. (D) Body weight measured on P10 and P21 $(+/+n=7-20, Q /+n=7-8) .(E)$ Representative voltage traces from a neuron injected with 100 pA current. Scale bar applies to all traces. (F) Input-output relationship generated for each injected current step $(+/+n=3$ mice, 20 cells, $Q /+n=3$ mice, 14 cells). ${ }^{*} P<0.05$ (F $(15,512)=$ 6.301, 2-way ANOVA with Sidak's multiple comparison. (G) Input resistance. ${ }^{* *} P<0.005(\mathrm{t}=3.22$, $\mathrm{df}=31) .(\mathbf{H})$ Rheobase. ${ }^{* * *} P<$ $0.0001(\mathrm{t}=5, \mathrm{df}=31)$, unpaired $t$ test. Data are represented as mean \pm SEM. assess the efficacy of Scn $2 a$ ASO administration commencing at a later stage of disease development. Since Q/+ mice have spontaneous seizures at P1 and mortality was first observed at P13, Scn $2 a$ ASO ED EO was administered at the upper limit of P14-P16. We found that the life span of $\mathrm{Q} /+$ mice was still significantly extended with the delayed therapeutic intervention. Moreover, in this treatment regime, Scn $2 a \mathrm{ASO}^{\mathrm{ED}} \mathrm{D}_{50}$ extended median survival to P111 ( $n=15$, Figure $3 \mathrm{C}$ ). The median survival for control ASO was P23 ( $n=8$, Figure $3 \mathrm{C})$. These data strongly indicate that later therapeutic intervention with $\operatorname{Scn} 2 a$ ASO still has compelling disease reversing capability.

Once it was established that Scn2a ASO treatment significantly extended survival, we tested its seizure-modifying ability. After Scn $2 a$ ASO administration at $\mathrm{P} 1, \mathrm{Q} /+$ mice were placed under 24-hour video monitoring at P21, and later at P30. Seizures above
Racine score 4 were counted (18). During P21 video monitoring, the control ASO group had a total of 21 seizures ( $n=8$ mice), while only 1 seizure was observed in the $\operatorname{Scn} 2 a \mathrm{ASO}_{\mathrm{ED}}$ group $(n=16$ mice). In the P30 video monitoring, 1 seizure was observed in the Scn $2 a$ ASO ED $_{50}$ group ( $n=17$ mice), while no mice survived in the control ASO group.

Since not all seizures have behavioral manifestation, 24-hour electrocorticography $(\mathrm{ECoG})$ recordings were performed. No electrographic seizures were detected in Scn $2 a$ ASO ED $_{50} \mathrm{Q} /+$ mice recorded at $\mathrm{P} 40-\mathrm{P} 45$. Furthermore, the number of spikes recorded on ECoG was similar to age-matched, untreated +/+ mice (Figure 4, A and B). When the ECoG was repeated at P56$\mathrm{P} 78$, inter-ictal spikes were significantly increased compared with earlier $\mathrm{P} 40-\mathrm{P} 45$ recordings, and spontaneous seizures were detected (Figure 4, C-E). 
A

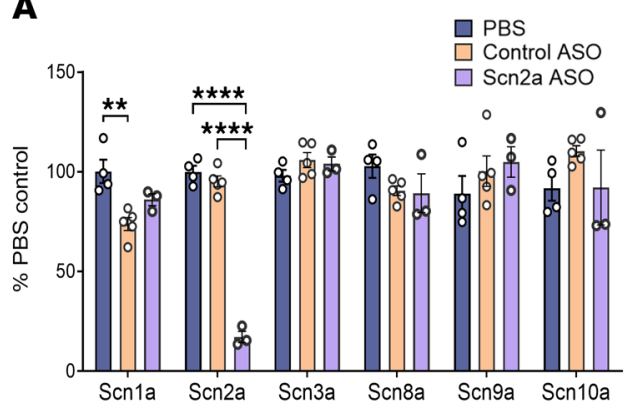

C
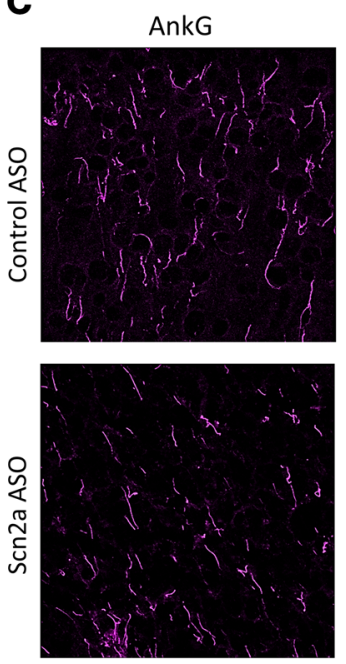

B

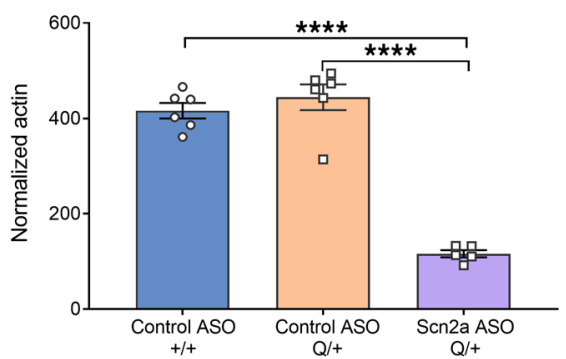

Collated
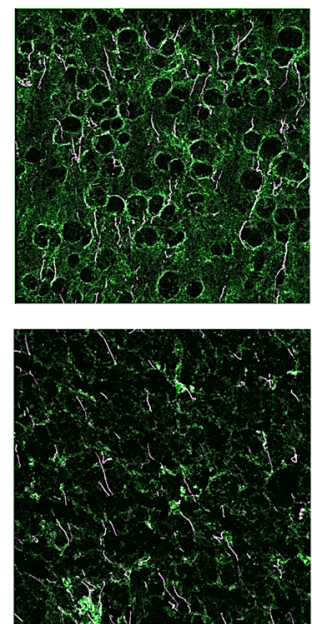

Figure 2. Scn2a mRNA and protein expression were reduced in mice $i . c . v$. injected with Scn2a ASO ED ${ }_{80}$ at P1. (A) Percentage of voltage-gated sodium channel isoform mRNA remaining in the cortex. $n=3-5+/+$ mice for each treatment group. ${ }^{* * *} P<$ $0.0001(\mathrm{~F}(2,9=212.6)),{ }^{* *} P<0.01(\mathrm{~F}(2,9$ $=10.30)$ ), 1-way ANOVA with Tukey's multiple comparison. (B) Level of Scn2a protein normalized to actin as determined by mass spectrometry, $n=5$ to 6 mice for each treatment group. ${ }^{* * *} P<0.0001(\mathrm{~F}(2$, $14=79.80))$, 1-way ANOVA with Tukey's multiple comparison. (C) Z-stack images of cortical slices labelled by AnkG (magenta) and Scn2a protein (green). One independent experiment, $n=2-3+/+$ mice for each treatment group. Scale bar: $20 \mu \mathrm{m}$. Data are represented as mean \pm SEM.
To determine the basis underlying seizure phenotype reemergence, we investigated the time course of Scn $2 a$ mRNA

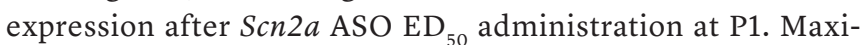
mum $\operatorname{Scn} 2 a$ reduction $(53.70 \% \pm 2.82 \%)$ was observed at $\mathrm{P} 15$, which was then decreased to $39.45 \% \pm 2.62 \%$ and $38.83 \% \pm$ $4.65 \%$ at $\mathrm{P} 30$ and $\mathrm{P} 45$ respectively (Figure $4 \mathrm{~F}$ ). At P60 when seizure features reappeared in ECoG, Scn $2 a$ mRNA was only reduced by $5.46 \% \pm 3.89 \%$ (Figure $4 \mathrm{~F}$ ). A simple linear regression was fitted from P15-P60 (equation $y=0.9849 \times-70.86$, $\mathrm{R}^{2}=0.7361$ ), and the time point at which $\operatorname{Scn} 2 a$ mRNA suppression was halved was $\mathrm{P} 45$. This strongly indicates that disease reemergence in $S c n 2 a$ ASO $\mathrm{ED}_{50}$-treated Q/+ mice correlated with an increase in $S c n 2 a$ expression from the initial knockdown levels.

Behavioral phenotype of Scn $2 a$ ASO ED $D_{50}$-treated $Q /+$ mice. Voltage-gated sodium channels are critical for early rodent neurodevelopment, and nonselective sodium channel blockers such as phenytoin are known to cause neurotoxicity when administered to neonatal mice (19). Thus, a battery of behavioral tests was performed in Q/+ mice treated with Scn $2 a$ ASO (Figure 5A). As no untreated $\mathrm{Q} /+$ mice survived after $\mathrm{P} 30$, comparisons in behavioral tests had to be made to age- and sex-matched untreated $+/+$ mice, instead of to the control ASO group.

The Q/+ mice i.c.v. injected with Scn $2 a \mathrm{ASO}^{\mathrm{ED}} \mathrm{D}_{50}$ at P1 had body weights similar to $+/+$ mice (Figure $5 \mathrm{~B}$ ). Looking at motor function, the $\operatorname{Scn} 2 a$ ASO $\mathrm{ED}_{50}$-treated Q/+ mice had limb muscle strength (Figure $5 \mathrm{C}$ ) and number of foot faults similar to $+/+$ mice on grid walk test (Figure 5D). The Scn $2 a$ ASO ED $_{50}$ treatment at P1 had no impact on the ambulatory time measured in locomotor assay (Figure 5E).

We further assessed the impact of Scn2a ASO on social function. In the 3-chamber social interaction test, Scn2a ASO $\mathrm{ED}_{50}{ }^{-}$ treated $\mathrm{Q} /+$ mice spent equivalent time interacting with a novel mouse as $+/+$ mice, indicating normal social approach (Figure $5 F)$. Interestingly, on the elevated plus maze, $\operatorname{Scn} 2 a \operatorname{ASO}_{50} \mathrm{Q} /+$ mice spent more time in the open arm and less time in the closed arm when compared with $+/+$ mice (Figure $5 G$ ).

Scn2a ASO ED ${ }_{80}$ shows higher efficacy but also changes multiple behaviors. We next investigated whether increasing ASO dose to cause an $80 \% \operatorname{Scn} 2 a$ reduction ( $\operatorname{Scn} 2 a$ ASO $\mathrm{ED}_{80}$ ) would further improve survival and seizure outcomes. Scn $2 a \mathrm{ASO}_{\mathrm{ED}}{ }_{80}$ was determined to be $10 \mu \mathrm{g}$ from dose-response curves for $\mathrm{P} 1 \mathrm{administration}$ (Supplemental Figure 3A). Q/+ mice were i.c.v. injected with Scn2a ASO $\mathrm{ED}_{80}$ at $\mathrm{P} 1$, and median survival was increased to P86 with this higher dose (Figure 6A). To monitor seizures, 24-hour video monitoring was performed. At P21 no seizures were observed ( $n$ $=10$ mice) and at P30 only 1 seizure was observed ( $n=13$ mice). Consistently, ECoG found similar spikes between Scn $2 a$ ASO ED ${ }_{80}$ $\mathrm{Q} /+$ mice and age-matched, untreated $+/+$ mice (Figure 6B). This indicates that the efficacy of Scn $2 a$ ASO is dose dependent.

The body weights of $+/+$ mice and $\operatorname{Scn} 2 a$ ASO ED $_{80}$ treated $\mathrm{Q} /+$ mice were similar as measured at P30 (Figure 6C). However, $\operatorname{Scn} 2 a$ ASO $\mathrm{ED}_{80}$ resulted in motor function changes that were not noted in the $\mathrm{ED}_{50}$ group. While the limb strength of 
A

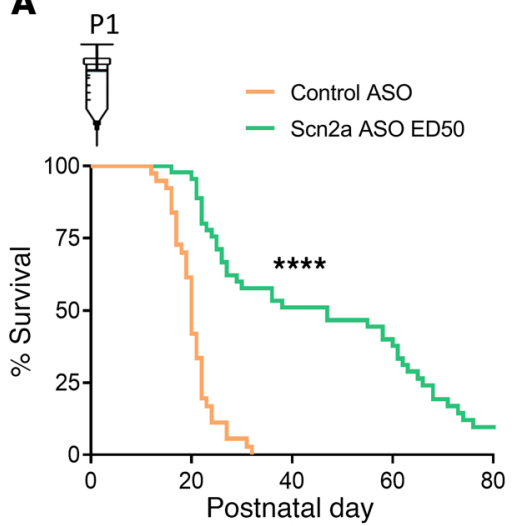

B

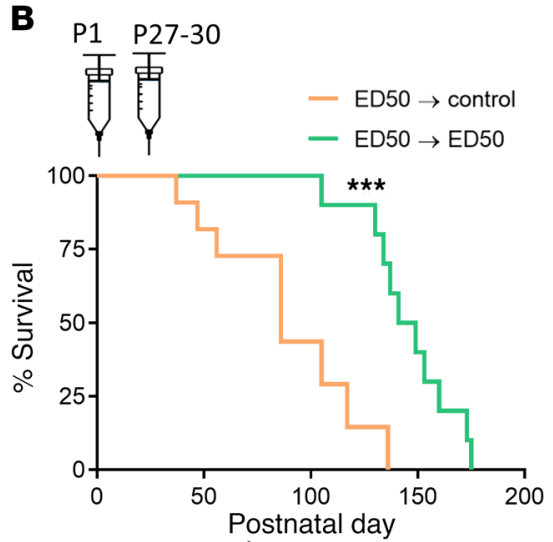

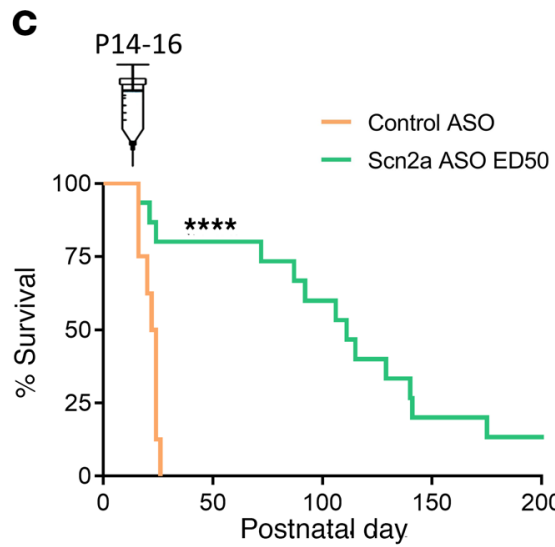

Figure 3. Scn2a ASO ED ${ }_{50}$ extends survival of Q/+ mice. (A) Survival curves of Q/+ mice i.c.v. injected with Scn2a ASO ED ${ }_{50}$ or the negative control ASO at P1. Control ASO (50 $\mu \mathrm{g}, n=39)$, Scn2a ASO ED 50 ( $2 \mu \mathrm{g}, n=49)$. (B) Survival curves of Q/+ mice i.c.v. injected with Scn2a ASO ED 50 (2 $\mu \mathrm{g})$ at P1, then redosed

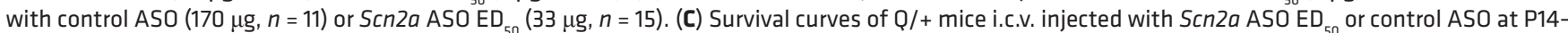
P16. Control ASO $(85 \mu \mathrm{g}, n=8)$, Scn2a ASO ED $50(20 \mu \mathrm{g}, n=15)$. Syringes indicate time of i.c.v. injection. ${ }^{* * *} P<0.0001,{ }^{* * *} P<0.005$, log rank test.

Scn $2 a \mathrm{ASO} \mathrm{ED}_{80} \mathrm{Q} /+$ mice were similar to +/+ mice (Figure 6D) on the grid walk test, $\operatorname{Scn} 2 a \mathrm{ASO}_{\mathrm{ED}} \mathrm{Q} /+$ mice had more foot faults than untreated $+/+$ mice (Figure $6 \mathrm{E}$ ). They also showed higher ambulatory time in the locomotor assay (Figure 6F). The Scn $2 a \mathrm{ASO} \mathrm{ED}_{80} \mathrm{Q} /+$ mice also spent less time in the closed arm and more time in the open arm of the elevated plus maze (Figure $6 \mathrm{G}$ ), and the magnitude of change was greater than in $S c n 2 a$ ASO $\mathrm{ED}_{50} \mathrm{Q} /+$ mice. Taken together, these data indicate that while the $\mathrm{ED}_{80}$ dose resulted in better survival outcomes than the $\mathrm{ED}_{50}$ dose, it also caused more behavior changes.

Tolerability of Scn2a ASO ED $D_{50}$ in neonatal +/+ mice. As part of the preclinical safety assessment, the tolerability of Scn2a ASO $\mathrm{ED}_{50}$ was examined in $+/+$ mice. It is critical to understand the safety of Scn2a ASO in the absence of gain-of-function variant, as $S c n 2 a$ depletion in early rodent development is detrimental (20, 21). Consistently, administering Scn $2 a \mathrm{ASO} \mathrm{ED}_{80}$ into P1+/+mice resulted in early mortality and smaller body weight when compared with the control ASO group (Supplemental Figure 4). Early lethality and body weight reduction were not observed when the Scn2a ASO dose was lowered to $\mathrm{ED}_{50}$ (Figure 7A).

The same behavioral studies performed on $\mathrm{Q} /+$ mice were repeated in +/+ mice i.c.v. injected with $S c n 2 a \mathrm{ASO} \mathrm{ED}_{50}$ or control $\mathrm{ASO}$ at P1. The $S c n 2 a$ ASO ED ${ }_{50}$ treatment at P1 did not alter limb muscle strength (Figure 7B), ambulatory time in locomotor assay (Figure 7D), or social approach (Figure 7E) in +/+ mice. However, Scn2a ASO ED ${ }_{50}$ caused higher number of foot faults on the grid walk test in $+/+$ mice (Figure $7 \mathrm{C}$ ). It should be noted that deficits in the grid walk assay were not observed for $\mathrm{Q} /+$ mice administered ASOs at $\mathrm{ED}_{50}$, suggesting that a lower side effect profile may occur in the disease context. As observed previously in Scn2a ASOtreated Q/+ mice, $\operatorname{Scn} 2 a \mathrm{ASO} \mathrm{ED}_{50}+/+$ mice also spent less time in the closed arm of the elevated plus maze (Figure $7 \mathrm{~F}$ ).

\section{Discussion}

Patients with the early seizure onset DEE caused by SCN2A gainof-function variants have poor clinical outcomes, indicating an urgent need for novel treatments. The correlation between genetic variants, functional effect, and clinical phenotype is well defined
$(11,12,16)$, making $S C N 2 A$ a credible candidate to trial ASO therapy to downregulate its expression. In this study, we demonstrated therapeutic benefits of ASO-mediated Scn $2 a$ mRNA and protein downregulation in a mouse model carrying one of the most recurrent $S C N 2 A$ gain-of-function DEE variants. Critically, the $\operatorname{Scn} 2 a$ ASO $\mathrm{ED}_{50}$ treatment prolonged life span in the diseased mice and rescued the seizure phenotype when given at P1. With detailed testing of $S c n 2 a$ ASO injection regimens, we showed that repeated administration provided better survival outcomes. In addition, ASO treatment at a later stage of disease development was also beneficial. These results are pertinent, as multiple dosing and therapy commencement after disease onset are the most likely scenarios expected in the clinical setting. Last, given the critical role of SCN2A in neurodevelopment and the fact that its loss of function has been linked to different disease phenotypes, we explored the therapeutic window of ASO-induced Scn2a downregulation. We identified that at the $\mathrm{ED}_{50}$ dose, efficacy was achieved with limited behavioral side effects in $\mathrm{Q} /+$ mice. The $\mathrm{ED}_{80}$ dose achieved higher efficacy in terms of survival and seizure suppression but resulted in more behavioral changes in $\mathrm{Q} /+$ mice.

Following 30 years of development, ASO technology has gained increasing recognition with the recent clinical success of ASO treatments for spinal muscular atrophy and Duchenne muscular dystrophy $(5,6)$. Recently, the ASO approach has demonstrated compelling efficacy in animal models of SCN1A and $S C N 8 A$ epilepsies $(22,23)$. The ASO approach has several advantages over other gene therapy approaches. First, the introduced change does not affect genetic information carried in the DNA but targets the intermediate mRNA molecules and the resulting protein production, circumventing potential irreversible off-target changes to the patient genome. Second, the effect of ASO on RNA is transient and any potential adverse effects can be stopped simply by refraining from further ASO administration. In this regard, an alternative strategy currently in development is to administer a fully complementary ASO decoy to neutralize the targeting ASO (24). Further, the effect of ASO treatment is dose dependent and can be adjusted, enabling identification of a specific therapeutic window for each target and/or patient. Finally, ASOs show differ- 
A

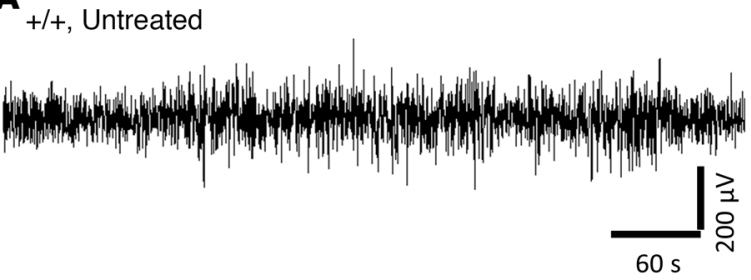

$\mathrm{Q} /+, \operatorname{Scn} 2 \mathrm{a}$ ASO ED50, P40-45

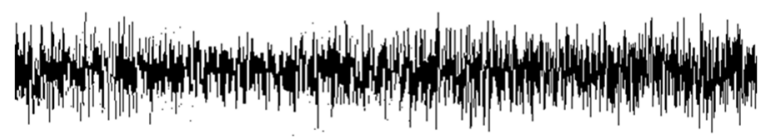

C

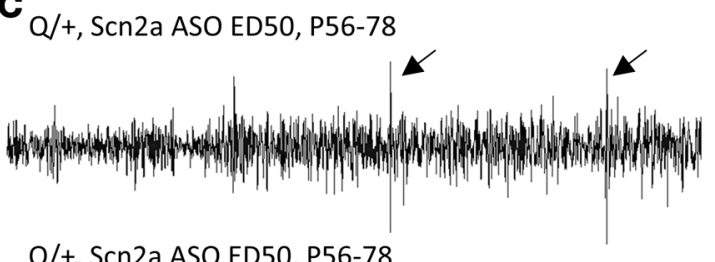

$\mathrm{Q} /+, \operatorname{Scn} 2 \mathrm{a}$ ASO ED50, P56-78

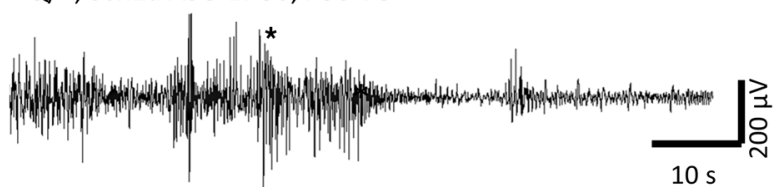

$10 \mathrm{~s}$
B

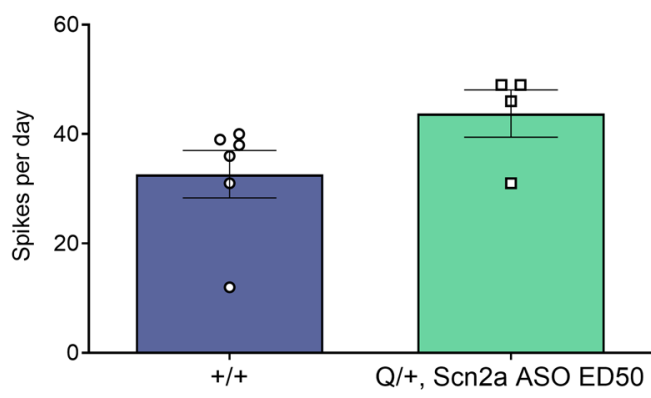

D

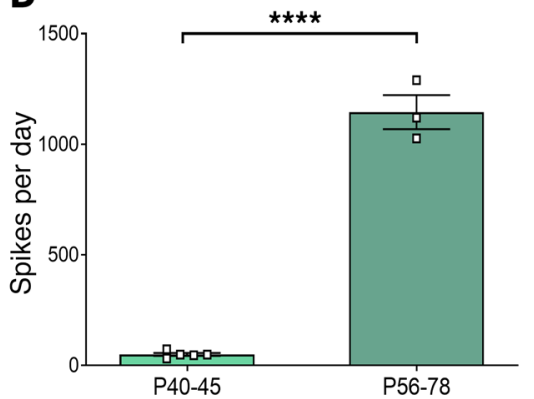

E

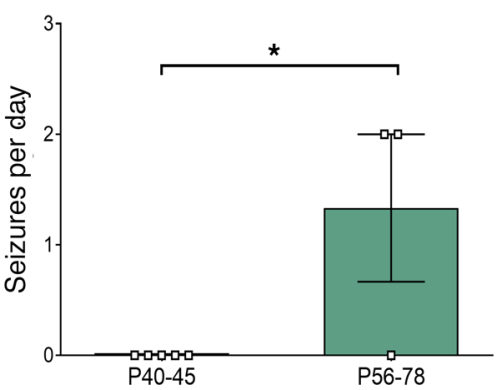

F $\square \mathrm{PBS}$

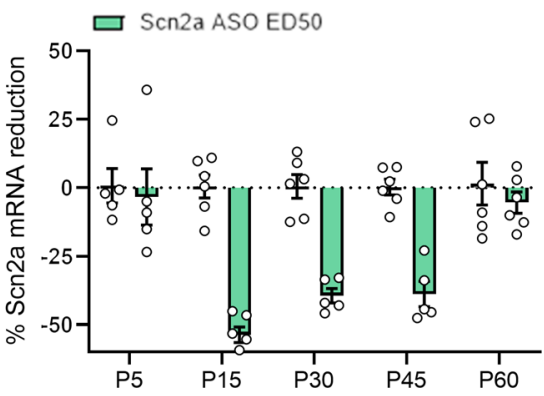

Figure 4. Scn2a ASO ED 50 suppresses seizure phenotype of Q/+ mice. (A) Representative ECoG traces recorded at P40-P45. Scale bar applies to all traces. (B) Number of spikes during 24-hour ECoG recorded at P40-P45 (+ $++n=6$, Scn2a ASO ED 50 / $+n=4)$. (C) Representative ECoC traces recorded at P56P78 in Q/+ mice treated with Scn2a ASO ED 5 at P1. Top panel: arrows indicate inter-ictal spikes. Bottom panel: seizure followed by post-ictal depression recorded on ECoG. Trace in box showed time expanded view of spike and seizure. (D and E) Number of spikes (D) or seizures (E) determined at different time points in Q/+ mice i.c.v. injected with Scn2a ASO ED ${ }_{50}$ at P1 (P40-P45 $\left.n=5, P 56-P 78 n=3\right) .{ }^{* *} P<0.005(t=19.25, d f=6),{ }^{*} P<0.05(t=2.739, \mathrm{df}=$ 6), unpaired 2 tailed $t$ test. (F) Time course of Scn2a mRNA reduction in mice i.c.v. injected with $\operatorname{Scn}_{2} \mathrm{ASO}_{\mathrm{A}} \mathrm{ED}_{50}$ at $\mathrm{P} 1, n=5-6$ per group per time point. Data are represented as mean \pm SEM.

ent modes of action, which can either affect the stability of mRNA or its splicing and interactions with other molecules (8).

To provide evidence that an ASO is a viable therapeutic strategy for $S C N 2 A$ gain-of-function DEEs, we generated a knockin mouse model carrying a recurrent gain-of-function $S C N 2 A$ variant linked to early seizure onset DEE. Compared with other existing SCN2A gain-of-function mouse models $(25,26)$, the $\mathrm{Q} /+$ mouse model has a severe disease phenotype, presenting with spontaneous seizures and premature death, with all untreated mice dying before P30. Summation of all available data indicate our model recapitulates the severe and early seizure phenotype of $S C N 2 A$ gain-of-function disease.

Despite the disease severity, a single bolus of Scn2a ASO
$\mathrm{ED}_{50}$ administered at $\mathrm{P} 1$ was able to extend survival and reduce seizure frequency of $\mathrm{Q} /+$ mice. This effect was correlated with ASO-mediated Scn $2 a$ downregulation at both the mRNA and protein levels. We demonstrated that the seizure phenotype reemerges when ASO is cleared from the brain over 60 days, which leads to the increase in Scn2a mRNA levels. A previous study showed that the in vivo half-life of ASO is several months (24). In the present study, the half-life of ASO is likely shorter, as it was administered at P1 and the rodent brain underwent rapid volume increase during development (27), thereby diluting the concentration of ASO in brain. Nevertheless, the seizuresuppressing effect of a single $\operatorname{Scn} 2 a$ ASO dose is still long-lasting, suggesting only infrequent dosing may be required in patients. 
A

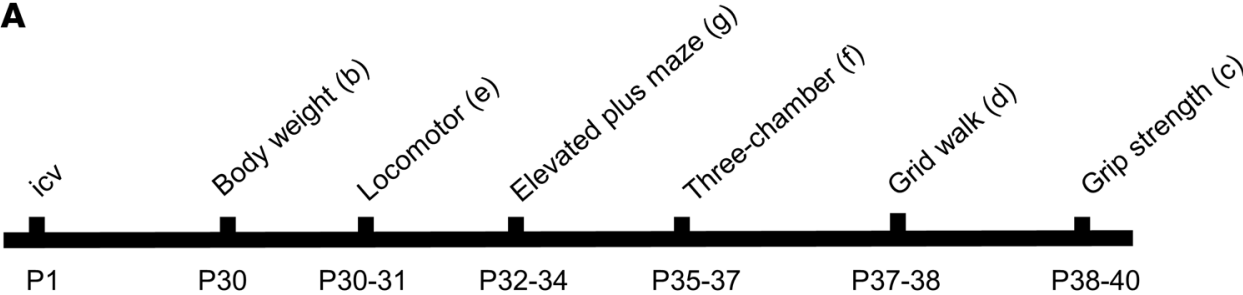

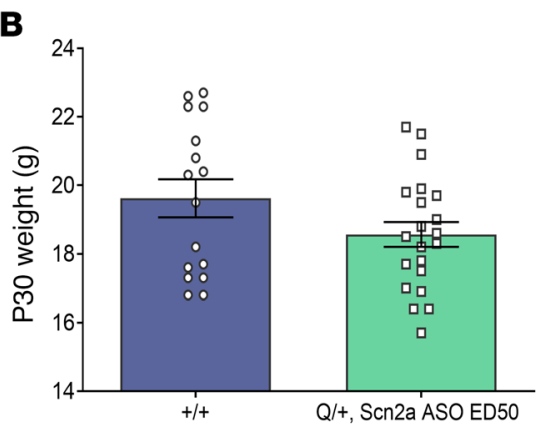

C
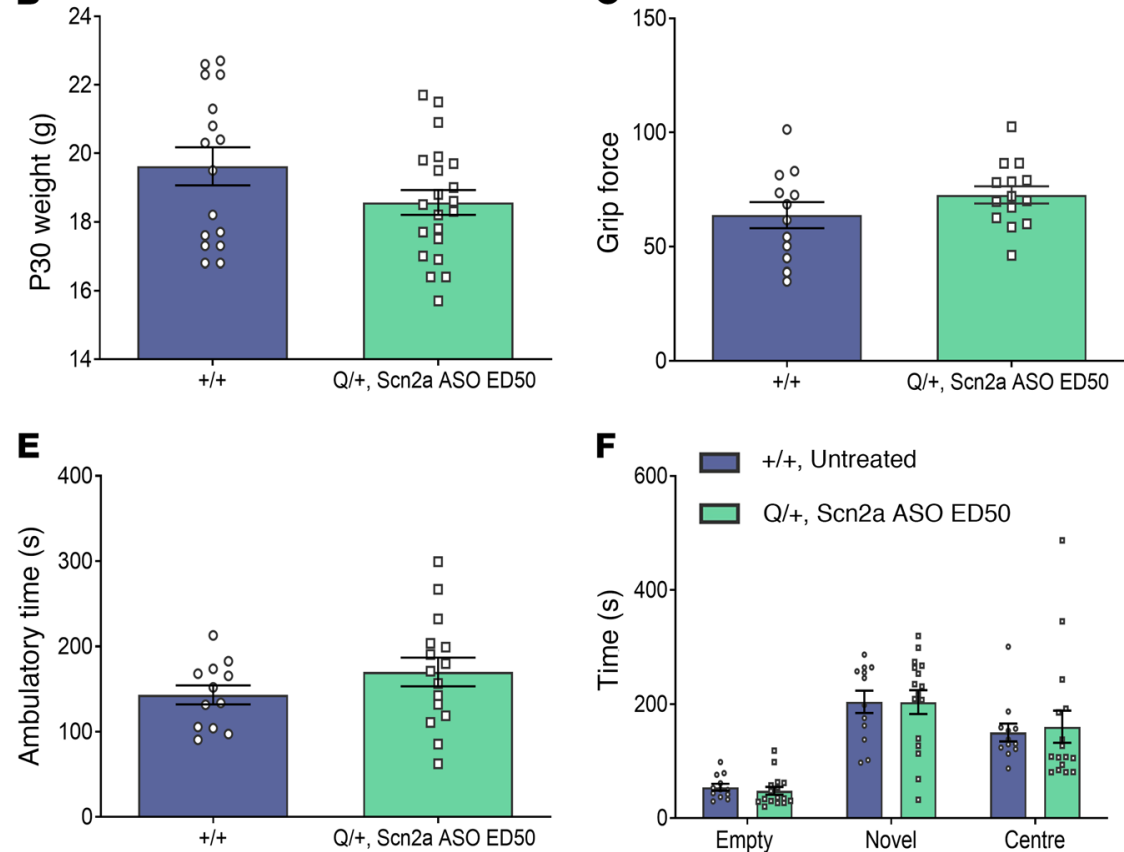

$\mathbf{F}$

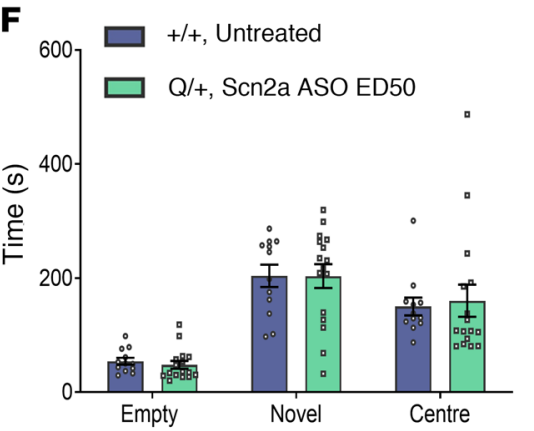

D
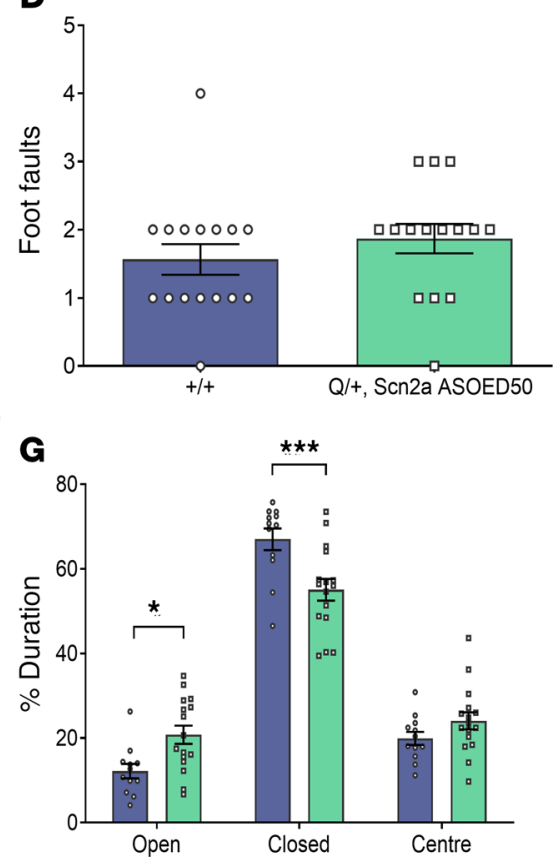

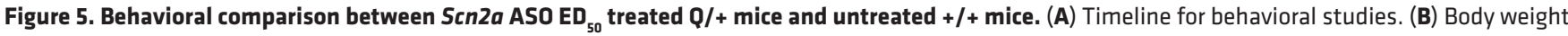
measured at P30. (C) Grip force. (D) Number of foot faults in grid walk test. (E) Ambulatory time measured in locomotor chamber. (F) Time spent in the different compartments of 3 -chamber social interaction test. (C) Duration spent in different arms of the elevated plus maze. ${ }^{* * *} P<0.001,{ }^{*} P<0.05$ (F (2, $78)=11.94)$, 2-way ANOVA with Sidak's multiple comparison, $n=10-21$ per genotype. Data are represented as mean \pm SEM.

Importantly, redosing at a later time point resulted in further protection from the severe seizure phenotype. It is noteworthy that reemergence of pathology that can be rescued by additional ASO treatment indicates that the acute impact of the pathogenic variant must underlie some proportion of the DEE phenotype. Importantly, we also showed that administration of Scn2a ASO 2 weeks after disease onset in mice was able to extend survival and reduce seizure frequency.

Testing of different dosing regimens has important implications for clinical development. Our data suggest a therapeutic regime may resemble the one currently employed for Nusinersen where upon initial "loading," subsequent injections occur every 3 to 4 months (28-30). Last, as the ASO drug class cannot pass the blood-brain barrier, ASO therapies for neurological disorders such as DEE would likely be delivered clinically via intrathecal injections. Intrathecal injection has proven to be an effective route of ASO delivery and is a technique that can be safely performed in adults as well as children $(31,32)$.

Since $S C N 2 A$ is critical for early postnatal development, the safety of ASO mediated Scn $2 a$ downregulation was explored in this study. The therapeutic window of $\operatorname{Scn} 2 a$ ASO depends on the disease state, that is, whether there is $S c n 2 a$ gain-of-function. This was demonstrated by the contrasting effect of $\operatorname{Scn} 2 a$ ASO ED $_{80}$ in $\mathrm{Q} /+$ and in +/+ mice, where the same treatment prolonged survival in $\mathrm{Q} /+$ mice but caused early mortality in $+/+$ mice. This suggests that a $\operatorname{Scn} 2 a$ gain-of-function variant would increase the tolerability to excessive $S c n 2 a$ reduction.

Due to premature death and frequent spontaneous seizures, the behavioral profile of untreated Q/+ mouse model could not be ascertained. Despite these limitations, we were able to show that $\mathrm{Q} /+$ mice administered with $S c n 2 a \mathrm{ASO}^{\mathrm{ED}} \mathrm{D}_{50}$ at P1 had a behavioral profile similar to untreated $+/+$ mice, with the exception in

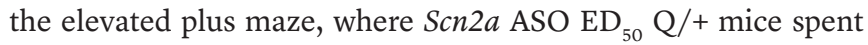
more time in the open arm and less time in the closed arm of the elevated plus maze. Importantly, this altered behavior on the elevated plus maze was also observed in $\operatorname{Scn} 2 a \mathrm{ED}_{50}+/+$ mice, which do not express the gain-of-function allele. Therefore, altered elevated plus maze behavior is unlikely part of the $S C N 2 A$ gainof-function disease phenotype. Rather, it is likely attributed to exaggerated pharmacology caused by action against the intended target (33), in this case, Scn $2 a$ downregulation. Indeed, increased time in the open arm of the elevated plus maze was previously reported in mouse models of $\operatorname{Scn} 2 a$ haploinsufficiency $(34,35)$. Another aspect of exaggerated pharmacology was altered motor 
A

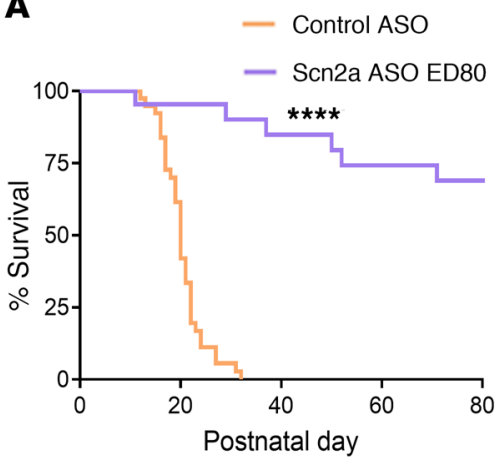

C

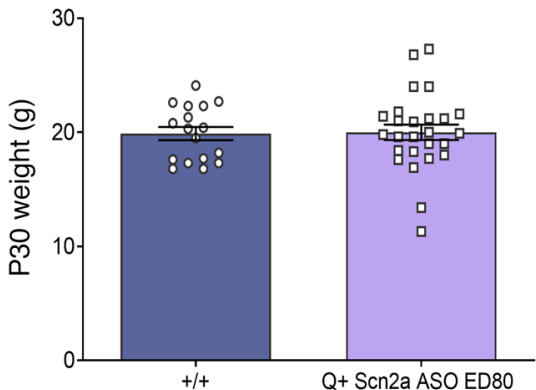

$\mathbf{F}$

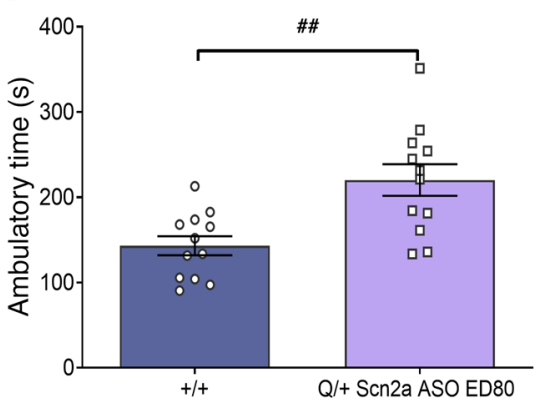

B

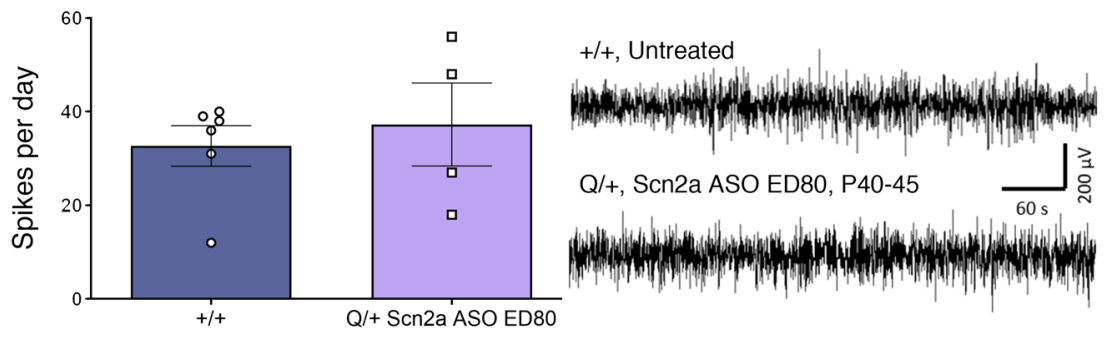

E
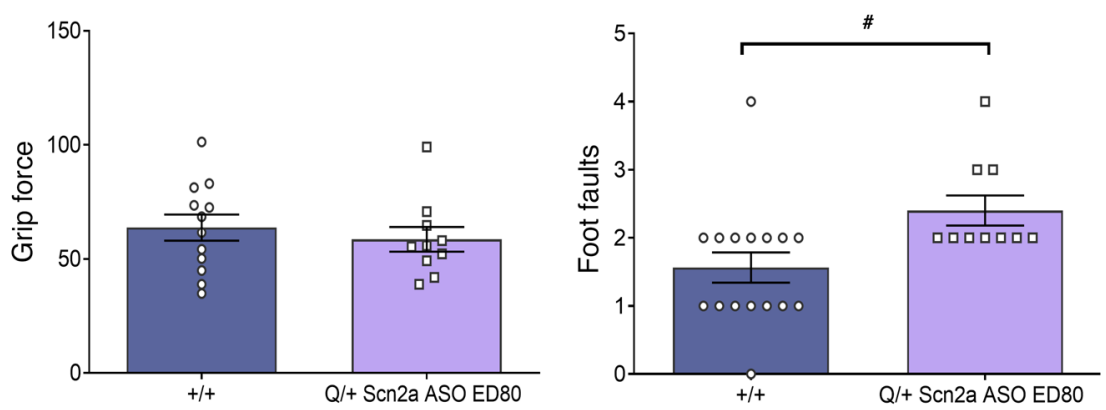

H

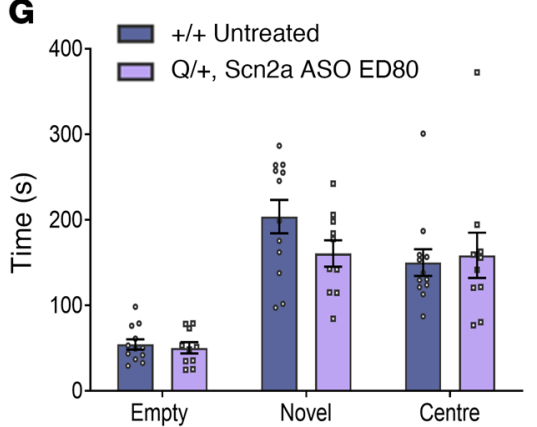

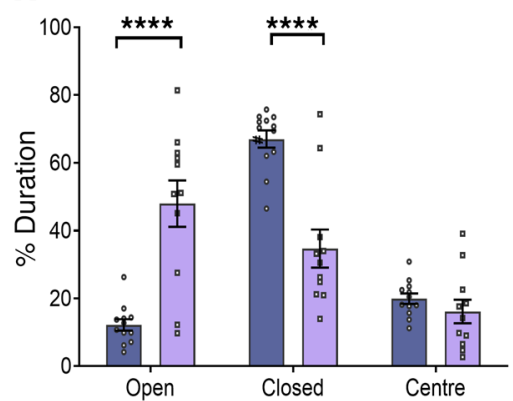

Figure 6. Effect of administering Scn2a ASO ED ${ }_{80}$ in Q/+ mice at P1. (A) Survival curves of Q/+ mice i.c.v. injected with $S_{C n 2 a} A S O E D{ }_{80}$ or the negative control ASO at P1. Control ASO $(50 \mu \mathrm{g}, n=39)$, Scn2a ASO ED 80 (10 $\mu \mathrm{g}, n=22)$. (B) Left: number of spikes during 24-hour ECoC recorded at P40-P45 (+/+n=6, Scn2a ASO ED 80 Q/+ n = 4). Right: representative ECoC traces. Scale bar applies to all traces. (C) Body weight measured at P30. (D) Grip force. (E) Number of foot faults in grid walk test. (F) Ambulatory time measured in locomotor chamber. (C) Duration spent in different arms of the elevated plus maze. $(\mathbf{H})$ Time spent in the different compartments of 3-chamber social interaction test. \#\#P<0.005 ( $\mathrm{t}=3.557, \mathrm{df}=22)$, \#P< $0.05(\mathrm{t}=2.518, \mathrm{df}=24)$, unpaired 2 tailed $t$ test. ${ }^{* * *} P<0.0001,(\mathrm{~F}(2,63)=36.72), 2$-way ANOVA with Sidak's multiple comparison, $n=10$ to 26 per genotype. Data are represented as mean \pm SEM.

function, such as increased foot faults on grid walk test in $\operatorname{Scn} 2 a$ $\mathrm{ED}_{50}+/+$ mice, which was not observed in $\operatorname{Scn} 2 a \mathrm{ED}_{50} \mathrm{Q} /+$ mice. The behavioral data again highlights that $S c n 2 a$ ASO exaggerated pharmacology is not only dose dependent, but also disease state dependent. Thus, a gain-of-function diagnosis is critical when considering $S c n 2 a$ downregulatory ASO as a therapy.

As it is clear that the therapeutic window of Scn $2 a$ downregulatory ASO would be limited by exaggerated pharmacology, one limitation in this study is that only $2 \operatorname{Scn} 2 a$ ASO doses were tested in $\mathrm{Q} /+$ mice. While $S c n 2 a \mathrm{ED}_{80}$ caused greater efficacy than $\mathrm{ED}_{50}$ in terms of extending life span, it also resulted in more behavioral changes, akin to $S c n 2 a$ haploinsufficiency. In contrast to $\mathrm{ED}_{80}$, the $\mathrm{ED}_{50}$ dose did not alter motor function, although it still increased the time $\mathrm{Q} /+$ mice spent on the open arm of the elevated plus maze. This rodent behavior has been interpreted as reduced anxiety or increased risk-seeking activity (36), but it is unclear if this is a direct correlate with human anxiety behavior. Ideally, a $\operatorname{Scn} 2 a$ ASO dose should be efficacious and without any signs of exaggerated pharmacology. Future preclinical study could achieve this by administering $\mathrm{Q} /+$ mice with a range of ASO doses below $\mathrm{ED}_{50}$, and assessing the survival, seizure, and behavioral outcomes at each lowered dose. The frequency of administration to maintain therapeutic level would also have to be explored. Our study already provides indirect evidence that $\mathrm{ED}_{40}$ is still of therapeutic benefit. In the experiment that tracked the time course of Scn2a ASO $\mathrm{ED}_{50}$ action from P1, Scn2a mRNA reduction was approximately $40 \%$ from P30-P45. We performed ECoG in Q/+ mice during that time period and detected no seizures or increases in inter-ictal spikes. 
A

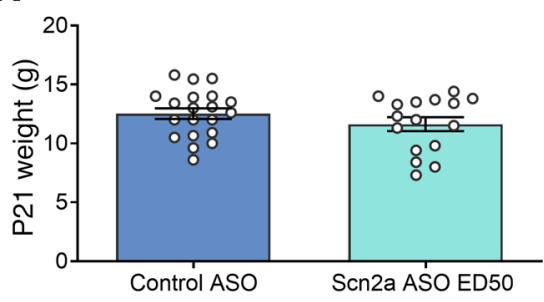

D

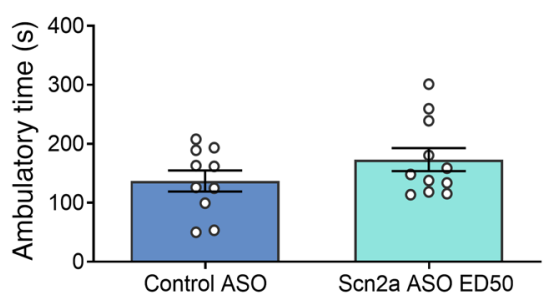

B

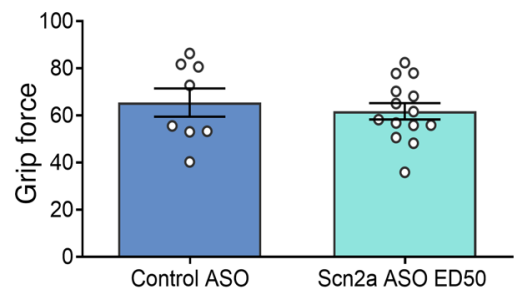

E

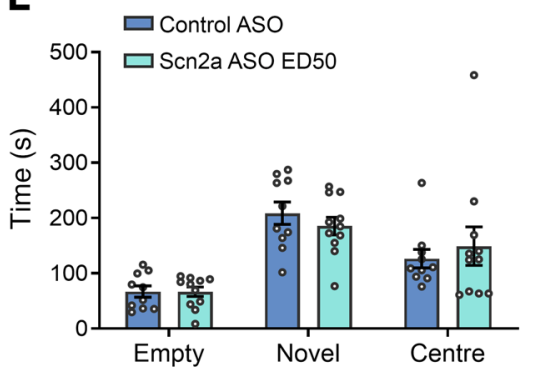

C

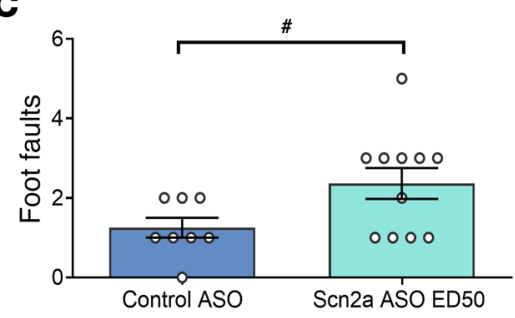

$\mathbf{F}$

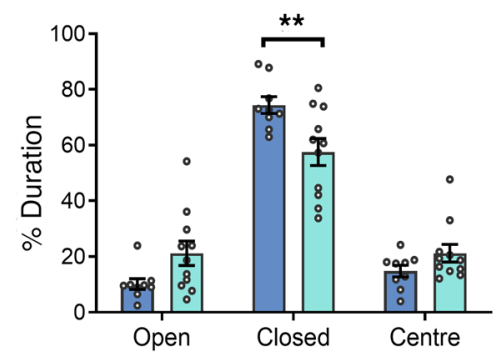

Figure 7. Effect of Scn2a ASO ED Ed $_{50}$ administration in P1 +/+ mice. (A) Body weight measured at P21. (B) Grip force. (C) Number of foot faults in grid walk test. $\# P<0.05(\mathrm{t}=2.207, \mathrm{df}=17)$, unpaired 2-tailed $t$ test. (D) Ambulatory time measured in locomotor chamber. (E) Time spent in the different compartments of 3 -chamber social interaction test. $(\mathbf{F})$ Percentage duration spent in different arms of the elevated plus maze. ${ }^{* *} P<0.01(F(5,54)=$ 8.512), 2-way ANOVA with Sidak's multiple comparison, $n=8$ to 20 per genotype. Data are represented as mean \pm SEM.

The clinical spectrum of SCN2A disorder continues to expand and many loss-of-function variants have been implicated in late seizure onset DEE and autism spectrum disorder. These patients would most likely not benefit from a SCN2A downregulatory ASO $(11,37)$. However, ASO technology can be used to upregulate gene and protein levels $(38,39)$, therefore also presenting an alternative therapeutic strategy for disorders caused by SCN2A loss-offunction variants.

In summary, this preclinical study demonstrated the therapeutic viability of ASO-mediated gene silencing for SCN2A gain-of-function disorders. The clinical development pathway for ASOs is well established and this study provides crucial efficacy and toxicology preclinical data for the SCN2A gain-offunction epilepsies that can be readily translated into clinical practice in the near future.

\section{Methods}

ASO preparation. We screened a collection of ASOs designed to target various regions of the mouse Scn2a mRNA. After screening a few hundred ASOs for their ability to reduce $\operatorname{Scn} 2 a$ levels in cultured mouse primary cortical neurons, the lead ASOs were i.c.v. injected into P1 $+/+$ mice. The most optimal ASO candidate was selected based on criteria as previously described (40). Selection criteria include parameters such as in vivo activity and tolerability. An ASO targeting mouse Scn $2 a$ (GCTCATGTTACTCCTACCCT) and a nontargeting control ASO (CCTATAGGACTATCCAGGAA) were used for the studies. Both ASOs were developed and synthesized by Ionis Pharmaceuticals. ASOs were synthesized as described (38) and were $20 \mathrm{bp}$ in length, with $5 \mathrm{MOE}$-modified nucleotides at each end of the oligonucleotide, and 10 DNA nucleotides in the center. The backbone of the ASOs consists of a mixture of phosphorothioate (PS) and phosphodiester (PO) linkages: 1-PS, 4-PO, 10-PS, 2-PO, and 2-PS (5' to $\left.3^{\prime}\right)$. ASOs were reconstituted with sterile $\mathrm{Ca}^{2+}$ and $\mathrm{Mg}^{2+}$ free PBS (Gibco).
Animal model. Male WT $(+/+)$ mice or mice heterozygous for Scn $2 a$ p.1883Q, equivalent to human pathogenic variant p.R1882Q $(\mathrm{Q} /+)$, were commercially generated on the C57/Bl6N background using the TurboKnockout technology (Cyagen). The point variant encoding p.R1883Q (CGG to CAG) was introduced into exon 26 in the 5' homology arm of the mouse Scn2a gene (NM_001099298.2). The targeting vector was generated by PCR using the BAC clone (Supplemental Figure 5). Within the targeting vector, the Neo cassette was flanked by LoxP sites, and diphtheria toxin A (DTA) was used for negative selection. The Neo cassette was engineered to be capable of self-deletion between the 2 LoxP sites without the need to breed to Flp deleter mice. This targeting vector was subsequently delivered into embryonic stem cells (ESCs) via electroporation. These ESCs were modified to generate $100 \%$ ESC-derived founder mice rather than chimeras, eliminating the need to screen for germline transmission. Targeting vector insertion was then confirmed by PCR screening, Southern blot, and sequencing. The targeted embryonic stem cell clones were then selected for blastocyst microinjection and implanted into surrogate dams. The F1 litters were used for experiments. The Scn $2 a$ knockout mouse model was generated on the C57/BL6J background (Australian National University) and contains a protein truncating variation, R851X. All animals were maintained in a temperature-controlled room, with a 12-hour light on/off cycle and free access to food and liquid.

Brain slice recording. Mice (P12-P14) were deeply anesthetized with $4 \%$ isoflurane, followed by brain tissue isolation. The brain was immediately transferred into ice-cold cutting solution consisting of $125 \mathrm{mM}$ Choline-Cl, $2.5 \mathrm{mM} \mathrm{KCl}, 0.4 \mathrm{mM} \mathrm{CaCl}, 6 \mathrm{mM} \mathrm{MgCl}_{2}, 1.25$ $\mathrm{mM} \mathrm{NaH}_{2} \mathrm{PO}_{4}, 26 \mathrm{mM} \mathrm{NaHCO}_{3}$, and $20 \mathrm{mM} \mathrm{D}$-glucose saturated with carbogen (95\% oxygen and 5\% carbon dioxide). Coronal sections $(300 \mu \mathrm{m})$ were sliced on a vibratome (VT1200, Leica). The brain slices were incubated in artificial cerebral spinal fluid consisting of $125 \mathrm{mM}$ $\mathrm{NaCl}, 2.5 \mathrm{mM} \mathrm{KCl}, 2 \mathrm{mM} \mathrm{CaCl}_{2}, 2 \mathrm{mM} \mathrm{MgCl}_{2}, 1.25 \mathrm{mM} \mathrm{NaH}_{2} \mathrm{PO}_{4}, 26$ 
$\mathrm{mM} \mathrm{NaHCO}$, and $10 \mathrm{mM}$ D-glucose saturated with carbogen for at least 1 hour at room temperature before recording.

Individual slices were placed in a recording chamber on an upright microscope (Slicescope Pro 1000, Scientifica) and perfused with aCSF at a rate of $2 \mathrm{~mL} /$ minute at $32^{\circ} \mathrm{C}$. Layer 2 or layer 3 excitatory neurons in the somatosensory cortex (S1) were identified with infrared-oblique illumination microscopy with a $40 \times$ objective lens (Olympus). Patch pipettes of 3 to $5 \mathrm{M} \Omega$ (Harvard Apparatus) were made using a puller (P-1000, Sutter Instruments) and were filled with internal solution consisting of $125 \mathrm{mM}$ K-gluconate, $5 \mathrm{mM} \mathrm{KCl}, 2 \mathrm{mM} \mathrm{MgCl}_{2}, 10 \mathrm{mM}$ HEPES, 4 mM ATP-Mg, 0.3 mM GTP-Na, 10 mM phosphocretine, 10 mM EGTA and pH to 7.3 with an osmolarity of 280 mOsm. Wholecell recording was made in current clamp mode using Axon Multiclamp 700B amplifiers (Molecular Devices). Data were acquired using pClamp v.10 software (Molecular Devices). Sampling frequency was $100 \mathrm{kHz}$ and low-pass Bessel was filtered at $10 \mathrm{kHz}$ (Digidata 1550, Molecular Devices). A holding current was injected to maintain membrane potential at approximately $-70 \mathrm{mV}$. Neuronal excitability was determined by measuring voltage during a series of $800 \mathrm{~ms}$ steps from -60 to $+300 \mathrm{pA}$ in $10 \mathrm{pA}$ increments every 2 seconds. Data were analyzed using the Axograph X software (Axograph).

Intracerebroventricular injection. P1 mice were cryo-anesthetized, then a Hamilton syringe (32 G Huber point) was inserted midway between lambda and right eye. A total $2 \mu \mathrm{L}$ injection containing PBS, control ASO, or Scn2a ASO was delivered at a depth of $2 \mathrm{~mm}$ below skin surface into the right ventricle. Pups were gently warmed until skin color returned to pink before being returned to home cage.

For mice over P14, i.c.v. injection was performed using stereotaxic apparatus (Kopf). Mice were anesthetized with $2 \%$ to $4 \%$ isoflurane and placed on a frame with the skull surface horizontal between lambda and bregma. A small piece of skull was removed by drilling, and an injection needle (30 G, PlasticsOne) was inserted at $0.25 \mathrm{~mm}$ posterior, $0.8 \mathrm{~mm}$ lateral to the bregma. The needle was lowered to a depth of 2.5 to $3 \mathrm{~mm}$ from brain surface. A total $5 \mu \mathrm{L}$ injection containing PBS, control ASO, or Scn2a ASO was delivered into the right ventricle using the syringe pump (KD Scientific) at a rate of $0.5 \mu \mathrm{L} / \mathrm{s}$. After 2-3 minutes, the needle was slowly withdrawn, and the incision was sutured.

Quantitative gene expression analysis (RT- $q P C R)$. Mice were i.c.v. injected with Scn $2 a$ ASO or control ASO $(10 \mu \mathrm{g})$ at P1 for the voltagegated sodium channel isoform qPCR (Figure 2A). Two weeks after i.c.v. injection, brains were harvested and snap-frozen in liquid nitrogen. Cortical tissue was homogenized and total RNA isolated using Trizol reagent according to the manufacturer's instructions (Invitrogen). Contaminating genomic DNA was removed with DNAse I treatment (Ambion/Life Technologies). RNA was assayed for quality and quantity using a NanoDrop 2000c Spectrophotometer (Thermo Fisher Scientific). For RT-qPCR, $1 \mu \mathrm{g}$ DNase-treated purified RNA was analyzed using a Taqman RNA to Ct kit process (Applied Biosystems) with a Taqman probe to each sodium channel gene in duplex with the mouse endogenous gene Glucuronidase Beta (Gusb). RT-qPCR was performed on the ViiA 7 Real-Time PCR System (Applied Biosystems) using cycling conditions specified by kit protocols (Supplemental Table 2). All RT-qPCR reactions were performed in triplicate. Relative gene abundance values were calculated by normalization to Gusb and referenced to the control groups using the $2 \Delta \Delta \mathrm{Ct}$ method.
For the dose-response curves (Supplemental Figure 3), mice were i.c.v. injected with a range of Scn2a ASO at P1, P15, or P30. Two weeks after i.c.v. injection, brains were harvested and snap-frozen in liquid nitrogen. Total RNA was isolated using the Trizol reagent according to the manufacturer's protocols (Thermo Fisher Scientific). Contaminating genomic DNA was removed with DNAse treatment (DNAfree Reagents, Ambion/Life Technologies). For RT-qPCR, oligo-dT primed cDNA was synthesized from 500 ng total RNA using Murine Moloney Leukemia Virus Reverse Transcriptase (Promega). RT-qPCR was performed on the ViiA 7 Real-Time PCR System, using SYBR green technology and GoTaq qPCR master mix (Promega) according to the manufacturer's protocols. The primers used for $\operatorname{Scn} 2 a$ detection were forward TGCTGTGCGGAAATCTGCC and reverse CGGATGCTCAAGAGAGACTGG. Relative gene expression values were obtained by normalization to the endogenous reference gene RPL32 (forward GAGGTGCTGCTGATGTGC, reverse GGCGTTGGGATTGGTGACT) and referenced to the control group using the $2 \Delta \Delta \mathrm{Ct}$ method.

Immunohistochemistry. To confirm the specificity of the antiScn $2 a$ protein antibody, brain sections were collected from PO Scn $2 a$ homozygous knockout (R851X) mice as negative controls (Supplemental Figure 2). As shown in Figure 2C, mice were i.c.v. injected with control ASO or Scn2a ASO $(10 \mu \mathrm{g})$ at P1, and brain tissue was isolated between P12-P15 and immediately frozen with liquid nitrogen. For Scn $2 a$ protein visualization, coronal sections $(10 \mu \mathrm{m})$ were cut on a cryostat (CM1850, Leica) and mounted on Superfrost plus microscope slides (VWR). After air-drying at room temperature for 30 minutes, sections were fixed in precooled acetone for 8 minutes. Sections were blocked for 1 hour in room temperature with Tris buffered saline supplemented with $3 \%$ goat serum and $0.3 \%$ Triton. Sections were then incubated with the following antibodies: polyclonal rabbit anti-AnkyrinG antibody (clone H-215, catalog sc28-561, Santa Cruz) at 1:1000, and monoclonal mouse anti-Scn $2 a$ protein (anti-Nav1.2 $\mathrm{Na}$ channel antibody, clone K69/3, catalog 75-024, Neuromab) at 1:500 overnight at $4^{\circ} \mathrm{C}$. After 5 washes, sections were incubated with fluorescently labelled secondary antibodies: donkey anti-rabbit-Alexa 647 at 1:800 (catalog A-31573, Invitrogen) and goat anti-mouse-Alexa 488 at 1:500 (catalog A11-001, Invitrogen) for 1 hour at room temperature. To identify the nuclei, sections were then stained with DAPI (4',6-Diamidine-2'-phenylindole dihydrochloride, catalog D9542, Sigma-Aldrich). Slides were covered with Prolong Gold Antifade (Invitrogen) and stored at $-30^{\circ} \mathrm{C}$. The $\mathrm{L} 2 / 3$ layer of somatosensory cortex was examined, and confocal images captured using a Zeiss Axio 780 upright microscope, using a PL-APO 40x/NA 1.4 oil objective and according to Nyquist criterion.

For ASO visualization (Supplemental Figure 1), mice were i.c.v. injected with ASO $10 \mu \mathrm{g}$ at P1 were perfused with $4 \%$ paraformaldehyde at P14. The brains were isolated and sequentially equilibrated in $10 \%$ to $30 \%$ sucrose (in $0.1 \mathrm{M} \mathrm{PB}$ ) at $4^{\circ} \mathrm{C}$. The brains were then embedded in OCT, frozen in chilled isopentane, and stored at $-80^{\circ} \mathrm{C}$ until use. Sagittal sections $(30 \mu \mathrm{m})$ were cut using a cryostat (CM1850, Leica) and mounted on positively charged slides. Sections were processed for immunohistochemistry to label neurons using NeuN (1:500; anti-NeuN antibody, clone A60, catalog ABN90, Merck Millipore), Calbindin (1:1000; mouse monoclonal, clone L109/57, catalog 75-448, UC Davis), and ASO backbone (1:7500; Ionis; ref. 17) with guinea pig and rabbit polyclonal primary antibodies. Tissue was incubated with primaries diluted in $5 \%$ normal goat serum and $0.15 \%$ Triton- $\mathrm{X}$ in 0.1 
M PB overnight and washed several times before incubating with secondary antibodies, donkey anti-guinea pig Alexa594 (catalog 20170, Biotium) and donkey anti-rabbit Alexa 647 (catalog A-31573, Invitrogen) for 2 hours at room temperature. Sections were then washed in 0.1 M PB, DAPI-stained (catalog D9542, Sigma-Aldrich) and mounted using ProLong Diamond antifade reagent (Thermo Fisher Scientific) and cover slipped. Confocal images were acquired using a Zeiss Axio 780 upright microscope. Mosaic and $z$-step images were taken with a PL-APO 20x/NA0.8 air objective and high-resolution images using the PL-APO 40x/NA 1.4 oil objective and according to Nyquist criterion. All images were then deconvolved using Huygens Essential (V15.10; Scientific Volume Imaging).

Quantification of ASO-positive neurons. For the quantification of ASO-positive neurons (Supplemental Table 1), the percentage of NeuN/ASO-positive cells was measured using ImageJ software (V1.53g). Images were acquired as outlined in immunohistochemistry (see above) and antibodies included ASO backbone, DAPI, NeuN for the neocortex, and calbindin-positive cerebellum Purkinje neurons, which are NeuN negative; Alexa Fluor 647, 594, and 488 dyes were used respectively as secondaries (Invitrogen).

Regions of interest (ROIs) were quantified across the whole brain at approximately $600 \mu \mathrm{m}$ intervals using serial-sectioned coronal brain slices. ROIs are summarized and shown in Supplemental Figure 1 and were determined using DAPI and NeuN labelling and based on regions outlined in the mouse brain atlas (41). At least 3 areas were sampled per ROI, per animal; area per image was $910 \mu \mathrm{m}^{2}$. For each image data set a maximum intensity projection was created from an optical $z$-stack of $20 \mu \mathrm{m}$ with a $2 \mu \mathrm{m}$ step size $(0.3 \mu \mathrm{m} /$ pixel xy) for ASO and neuron markers. Preprocessing included background subtraction and conversion to 8-bit binary image. NeuN positive cell count was obtained using analyze particles (size filter $35-300 \mu \mathrm{m}^{2}$ ) with an average of 8000 NeuN-positive cells analyzed across each mouse brain to confirm spatial overlap with the ASO fluorescence signal. A no primary control (NPC) image was also acquired to determine background signal for the ASO channel, and threshold was determined using the signal intensity profile. NPC images were acquired using the same confocal image settings, including laser power, gain, and digital offset, as those used for all experimental data $(n=4$ mice).

Mass spectrometry. Mice were i.c.v. injected with ASO (10 $\mu \mathrm{g})$ at P1 and brains were collected and snap frozen at P12-P15. Approximately $100 \mathrm{mg}$ cortical tissue was removed and added in a 1:5 ratio to extraction buffer, which consisted of $1 \times$ protease inhibitor, 1\% sodium deoxycholate, and $100 \mathrm{mM}$ TEAB (triethylammonium bicarbonate). Samples were vortexed and sonicated using the Bioruptor Plus (Diagenode) until completely homogenized. Protein concentration was determined using the bicinchoninic acid assay (BCA assay) (Thermo Fisher Scientific). Protein reduction was performed with DL-Dithiothreitol (DTT) to a final concentration of $5 \mathrm{mM}$, for 30 minutes at $60^{\circ} \mathrm{C}$. Samples were then alkylated in the dark with iodoacetamide (IAA) to a final concentration of $10 \mathrm{mM}$ for 30 minutes at $37^{\circ} \mathrm{C}$. Samples were quenched with additional DTT to a final concentration of 10 $\mathrm{mM}$ and incubated at $37^{\circ} \mathrm{C}$ for 30 minutes. The $\mathrm{pH}$ was checked for optimal trypsin digestion. A quantity of $100 \mu \mathrm{g}$ protein was added to Axygen Maxymum Recovery low-bind microcentrifuge tubes, followed by the addition of trypsin in a 1:40 ratio, and incubated at $37^{\circ} \mathrm{C}$ for 16 hours (overnight).
Deoxycholate was precipitated using $10 \%$ formic acid (FA). Samples were spiked with heavy peptide standard mix (JPT Peptide Technologies) and centrifuged at $12,000 \mathrm{~g}$ for 10 minutes to pellet the sodium deoxycholate. The supernatant was transferred to new lowbind tubes. Solid phase extraction clean up occurred using the AssayMAP Bravo Platform (Agilent) on C18 plates using the standard protocol from the manufacturer. Samples were dried down and stored in $-80^{\circ} \mathrm{C}$ before being resuspended in $2 \%$ acetonitrile, $0.05 \%$ trifluoroacetic acid to prepare for mass spectrometry (MS).

Agilent Technologies 6495 triple quadrupole LC-MS/MS was used for the relative quantification and detection of $\operatorname{Scn} 2 a$ protein and other target peptides. The method was optimized using Skyline software (MacCoss Lab, University of Washington) and Agilent Mass Hunter software. The transitions and method settings can be found in the supplementary material.

Spontaneous seizure monitoring. Q/+ mice were placed under 24-hour video monitoring at P21 and P30 in their home cage using a digital camera (EVO2, Pacific Communications). Videos were reviewed by 2 experimenters and seizures over Racine score 4 were noted (18).

Electrocorticography (ECoG). Mice (P30-P35) were anesthetized with $2 \%$ to $4 \%$ isoflurane and placed in a stereotaxic apparatus (Kopf) with the skull surface horizontal between lambda and bregma. Epidural electrodes, 1 reference and 2 recording (Pinnacle Technology), were placed on the brain surface. A ball of silver wire placed on top of the skull surface was used as a ground electrode. All electrodes were connected to a headmount (Pinnacle Technology) and secured to the skull with dental cement. Mice were allowed to recover for a minimum of 5 days before ECoG recording. Brain cortical activity was sampled at $250 \mathrm{~Hz}$ for 24 hours (Pinnacle Technology), with low pass and high pass filters of 40 $\mathrm{Hz}$ and $0.5 \mathrm{~Hz}$, respectively. During recording, mice could move freely in the cage with food and water ad libitum. ECoG data were analyzed using ClampFit 10.7 (Molecular Devices), and a spike was identified when the amplitude was 2.5 times baseline and duration was shorter than $80 \mathrm{~ms}$.

Behavioral tests. All behavioral experiments were performed during the light phase between the 10:00 and 17:00 hours, with a minimum of overnight recovery between tests. Mice were acclimatized to the testing rooms for at least 30 minutes before experimentation. All equipment was thoroughly cleaned and disinfected with $70 \%-80 \%$ ethanol between trials or testing of each mouse.

Locomotor test. Spontaneous locomotor activity was measured automatically with a laser-beam system monitoring distance and movements according to prescribed parameters (MedAssociates). Each mouse was placed in the center of the activity cage $(27 \times 27 \mathrm{~cm})$ for 30 minutes under dimmed lighting.

Grid walk test. Each mouse was placed on an elevated grid apparatus $(30 \mathrm{~cm})$ and allowed to freely walk along a $1 \mathrm{~m}$ grid of stainless steel bars ( $3 \mathrm{~mm}$ diameter) normally spaced $1 \mathrm{~cm}$ apart. The test was performed after 3 successful training runs. For the test, the grid bars in the last $50 \mathrm{~cm}$ were replaced with one of several set patterns that contain missing bars at a gap on 1 or 2 bars. Behaviors on the last $50 \mathrm{~cm}$ grid were recorded with a digital camera (EVO2, Pacific Communications). Videos were reviewed and the number of foot faults (total miss or deep slip) were noted (42).

Grip strength. A grip strength meter (Bioseb) was used to assess strength and control of the fore and hind paws. Mice were rested on the angled stainless-steel mesh assembly, grasping the mesh with all 4 
limbs. Mice were gently pulled by the tail away from the mesh, and the maximum force prior to release of the paws was recorded. Four trials were performed by 2 experimenters on each mouse.

Elevated plus maze. The base of the maze was custom made of Perspex containing 2 open arms $(7 \times 31 \mathrm{~cm})$ and 2 enclosed arms $(7 \times 31$ $\times 15 \mathrm{~cm})$ extending from a central platform $(5 \times 5 \mathrm{~cm})$ and was elevated $40 \mathrm{~cm}$ above the floor. At the beginning of the experiment, each mouse was placed in the center of the maze facing the enclosed arm. Trial duration was 10 minutes. The duration on the open and closed arms of the elevated plus maze was tracked by the TopScan software (Clever Sys Inc).

Three-chamber social interaction test. Mice were subjected to 3-chamber social test to assess social interaction behaviors. For mice i.c.v. injected at $\mathrm{P} 1$, the 3-chamber apparatus consisted of a Plexiglas rectangular box $(40 \times 38 \mathrm{~cm})$ divided into 3 compartments with 2 transparent walls. The 2 openings on the walls allowed free access into each of the 3 chambers. A wired cage of equal size $(16 \times$ $10 \mathrm{~cm})$ was placed in each of the 2 outside compartments $(16 \times 38$ $\mathrm{cm})$. The interaction zone was defined as $2.5 \mathrm{~cm}$ in front of the wired cage. Movement and duration were measured by TopScan software (Clever Sys Inc.). During habituation (5-10 minutes), the mouse was placed in the middle chamber and could freely explore all 3 chambers. An unfamiliar, age-, strain-, and sex-matched novel mouse was placed in the wired cage on the nonpreferred side, as determined from habituation. Activity of the test subject was then monitored for a further 10 minutes.

Data analysis. All data are presented as mean \pm SEM. Doseresponse curves were fitted with data points using Motulsky regression. All statistical analyses were performed by GraphPad Prism 7, and significance was determined when $P$ was less than 0.05 .
Study approval. All studies involving animals were carried out in accordance with the Guide for the Care and Use of Laboratory Animals (National Academies Press, 2011) and were approved by the Florey Institute Animal Ethics Committee.

\section{Author contributions}

ML, NJ, PJN, and LEB designed and carried out experiments, performed data analyses, and drafted the manuscript. B Rollo, $\mathrm{S}$ Pachernegg, A Sedo, and JH performed qPCR experiments and analyses. KR and A Sedo performed immunohistochemistry experiments and analyses. LD and LJ performed behavioral experiments and analyses. TB and B Roberts performed mass spectrometry experiments and analyses. A Soriano, AN, KD, SM, CAR, FR, and $S$ Petrou designed and coordinated the study. All authors read and contributed to the revision of manuscript.

\section{Acknowledgments}

We would like to thank Lynley Cordeiro for logistical and technical support for this project. This study was supported by National Health and Medical Research Council (NHMRC) program grant 10915693, NHMRC fellowship GNT1005050 (to S Petrou), RogCon Biosciences, and Praxis Precision Medicines.

Address correspondence to: Steven Petrou, Florey Institute of Neuroscience and Mental Health, 30 Royal Parade, Parkville, Victoria, 3052 Australia. Phone: 613.9035.3628; Email: steven. petrou@florey.edu.au.

B Roberts's present address is: Monash University, Clayton, Victoria, Australia.
1. Scheffer IE, et al. ILAE classification of the epilepsies: Position paper of the ILAE Commission for Classification and Terminology. Epilepsia. 2017;58(4):512-521.

2. Maljevic S, et al. Models for discovery of targeted therapy in genetic epileptic encephalopathies. J Neurochem. 2017;143(1):30-48.

3. Mulley JC, et al. Channelopathies as a genetic cause of epilepsy. Curr Opin Neurol. 2003;16(2):171-176.

4. Oyrer J, et al. Ion channels in genetic epilepsy: from genes and mechanisms to disease-targeted therapies. Pharmacol Rev. 2018;70(1):142-173.

5. Aartsma-Rus A, Krieg AM. FDA approves eteplirsen for duchenne muscular dystrophy: the next chapter in the eteplirsen saga. Nucleic Acid Ther. 2017;27(1):1-3.

6. Corey DR. Nusinersen, an antisense oligonucleotide drug for spinal muscular atrophy. Nat Neurosci. 2017;20(4):497-499.

7. Bennett CF. Therapeutic antisense oligonucleotides are coming of age. Annu Rev Med. 2019;70:307-321.

8. Bennett CF, Swayze EE. RNA targeting therapeutics: molecular mechanisms of antisense oligonucleotides as a therapeutic platform. Annu Rev Pharmacol Toxicol. 2010;50:259-293.

9. Liao Y, et al. Molecular correlates of age-dependent seizures in an inherited neonatal-infantile epilepsy. Brain. 2010;133(pt 5):1403-1414.

10. Gazina EV, et al. 'Neonatal' Nav1.2 reduces neuronal excitability and affects seizure susceptibility and behaviour. Hum Mol Genet. 2015;24(5):1457-1468.

11. Wolff M, et al. Genetic and phenotypic heterogeneity suggest therapeutic implications in SCN2A-related disorders. Brain. 2017;140(5):1316-1336.

12. Sanders SJ, et al. Progress in understanding and treating SCN2A-mediated disorders. Trends Neurosci. 2018;41(7):442-456.

13. Howell KB, et al. SCN2A encephalopathy: a major cause of epilepsy of infancy with migrating focal seizures. Neurology. 2015;85(11):958-966.

14. Carvill GL, et al. Targeted resequencing in epileptic encephalopathies identifies de novo mutations in CHD2 and SYNGAP1. Nat Genet. 2013;45(7):825-830.

15. Allen AS, et al. De novo mutations in epileptic encephalopathies. Nature. 2013;501(7466):217-221.

16. Berecki G, et al. Dynamic action potential clamp predicts functional separation in mild familial and severe de novo forms of SCN2A epilepsy. Proc Natl Acad Sci U S A. 2018;115(24):E5516-E5525.

17. Kordasiewicz HB, et al. Sustained therapeutic reversal of Huntington's disease by transient repression of huntingtin synthesis. Neuron. 2012;74(6):1031-1044.

18. Ihara Y, et al. Retigabine, a Kv7.2/Kv7.3-channel opener, attenuates drug-induced seizures in knock-in mice harboring Kcnq2 mutations. PLoS
One. 2016;11(2):e0150095.

19. Hatta T, et al. Neurotoxic effects of phenytoin on postnatal mouse brain development following neonatal administration. Neurotoxicol Teratol. 1999;21(1):21-28.

20. Planells-Cases R, et al. Neuronal death and perinatal lethality in voltage-gated sodium channel alpha(II)-deficient mice. Biophys J. 2000;78(6):2878-2891.

21. Ogiwara I, et al. Nav1.2 haplodeficiency in excitatory neurons causes absence-like seizures in mice. Commun Biol. 2018;1:96.

22. Lenk GM, et al. Scn8a antisense oligonucleotide is protective in mouse models of SCN8A encephalopathy and dravet syndrome. Ann Neurol. 2020;87(3):339-346.

23. Han Z, et al. Antisense oligonucleotides increase Scn1a expression and reduce seizures and SUDEP incidence in a mouse model of Dravet syndrome. Sci Transl Med. 2020;12(558):eaaz6100.

24. Rigo F, et al. Pharmacology of a central nervous system delivered 2'-O-methoxyethyl-modified survival of motor neuron splicing oligonucleotide in mice and nonhuman primates. J Pharmacol Exp Ther. 2014;350(1):46-55.

25. Kearney JA, et al. A gain-of-function mutation in the sodium channel gene Scn2a results in seizures and behavioral abnormalities. Neuroscience. 2001;102(2):307-317.

26. Schattling $\mathrm{B}$, et al. Activity of $\mathrm{Na}_{\mathrm{v}} 1.2$ promotes neurodegeneration in an animal model of multi- 
ple sclerosis. JCI Insight. 2016;1(19):e89810.

27. Semple BD, et al. Brain development in rodents and humans: Identifying benchmarks of maturation and vulnerability to injury across species. Prog Neurobiol. 2013;106-107:1-16.

28. Finkel RS, et al. Treatment of infantile-onset spinal muscular atrophy with nusinersen: a phase 2, open-label, dose-escalation study. Lancet. 2016;388(10063):3017-3026.

29. Chiriboga CA, et al. Results from a phase 1 study of nusinersen (ISIS-SMN(Rx)) in children with spinal muscular atrophy. Neurology. 2016;86(10):890-897.

30. Finkel RS, et al. Nusinersen versus sham control in infantile-onset spinal muscular atrophy. $N$ Engl JMed. 2017;377(18):1723-1732.

31. Mousa MA, et al. A comprehensive institutional overview of intrathecal nusinersen injections for spinal muscular atrophy. Pediatr Radiol. 2018;48(12):1797-1805.

32. Wurster CD, et al. Intrathecal administration of nusinersen in adolescent and adult SMA type 2 and 3 patients. J Neurol. 2019;266(1):183-194.

33. Kornbrust D, et al. Oligo safety working group exaggerated pharmacology subcommittee consensus document. Nucleic Acid Ther. 2013;23(1):21-28.

34. Spratt PWE, et al. The autism-associated gene Scn2a contributes to dendritic excitability and synaptic function in the prefrontal cortex. Neuron. 2019;103(4):673-685.

35. Tatsukawa T, et al. Scn $2 a$ haploinsufficient mice display a spectrum of phenotypes affecting anxiety, sociability, memory flexibility and ampakine CX516 rescues their hyperactivity. Mol Autism. 2019;10:15.

36. Walf AA, Frye CA. The use of the elevated plus maze as an assay of anxiety-related behavior in rodents. Nat Protoc. 2007;2(2):322-328.

37. Ben-Shalom R, et al. Opposing effects on NaV1.2 function underlie differences between SCN2A variants observed in individuals with autism spectrum disorder or infantile seizures. Biol Psychiatry. 2017;82(3):224-232.

38. Liang XH, et al. Translation efficiency of mRNAs is increased by antisense oligonucleotides targeting upstream open reading frames. Nat Biotechnol. 2016;34(8):875-880.

39. Liang XH, et al. Antisense oligonucleotides targeting translation inhibitory elements in 5' UTRs can selectively increase protein levels. Nucleic Acids Res. 2017;45(16):9528-9546.

40. Rigo F, et al. Antisense oligonucleotide-based therapies for diseases caused by pre-mRNA processing defects. Adv Exp Med Biol. 2014;825:303-352.

41. Franklin KBJ, Paxinos G. The Mouse Brain in Stereotaxic Coordinates. Academic Press; 2007.

42. Farr TD, et al. Bilateral alteration in stepping pattern after unilateral motor cortex injury: a new test strategy for analysis of skilled limb movements in neurological mouse models. J Neurosci Methods. 2006;153(1):104-113. 